\title{
ELECTRON-IMMUNOGYTOCHEMICAL LOCALIZATION OF LAMININ AND TYPE-IV COLLAGEN IN THE ENAMEL ORGAN OF THE RAT INCISOR
}

\author{
Takashi SAWADA ${ }^{1}$, TakaAki YanagisaWA ${ }^{1}$, Shosaburo TAKUMA $^{1}$, \\ HideAKI HASEGAWA ${ }^{2}$ and KeIIGHI WATANABE ${ }^{3}$ \\ Department of Oral Pathology ${ }^{1}$, Tokyo Dental College, Masago 1-2-2, Mihama-ku, Chiba-city, \\ Chiba 261, Cell Biology Research Laboratory ${ }^{2}$ and Department of Pathology ${ }^{3}$, \\ Tokai University School of Medicine, Isehara-city, \\ Kanagawa 259-11
}

Received for publication July 31, 1991 and in revised form November 6, 1991

\begin{abstract}
The presence of laminin and type-IV collagen in the basement membrane of the rat incisor enamel organ at different stages of odontogenesis was demonstrated by means of pre-embedding immunoperoxidase method.

Immunoreaction for laminin and type-IV collagen was observed in the basement membrane and in the fibrillar layer attached to the membrane demarcating the inner enamel epithelium from the dental papilla. Occasional reaction products for laminin and type-IV collagen localized in the rough endoplasmic reticulum cisternae in cells of the inner enamel epithelium and dental papilla indicated cooperative production of basement membrane components. The basement membrane of differentiating ameloblasts showed reactions for laminin and type-IV collagen, though the intensity was consistently lower than that of reactions in the inner enamel epithelium. These reactions gradually became indistinct and discontinuous and disappeared entirely prior to dentin formation. At this time, positive reaction products were observed in the deep, irregular invaginations and coated pits in the distal ends of differentiating ameloblasts, indicating resorption of basement membrane components. The basement membrane-like structure appearing between the maturing ameloblasts and the enamel surface reacted positively for laminin. Occasional occurrence of reaction products in rough endoplasmic reticulum of maturing ameloblasts indicated laminin production. No reaction products for type-IV collagen, however, occurred in the basement membrane-like structure. Positive immunoreactions were observed in the basement membrane demarcating the outer enamel epithelium and the papillary layer from the dental sac. Basement membranes of blood capillaries that had penetrated into the papillary layer also showed intense immunoreaction. Reaction products were frequently seen in the perinuclear spaces and the rough endoplasmic reticulum cisternae of outer enamel epithelium cells but not of papillary layer cells.
\end{abstract}

It has been suggested that the dental basement membrane separating the tooth enamel organ from mesenchymal tissues of dental papillae and dental sacs plays an important role in odontogenesis (4). The chemical composition, origin, and ultimate fate of this membrane, however, remain obscure.

Recent immunofluorescence studies $(9,26)$ have shown that basement membrane components (laminin and type-IV collagen) are also localized in the dental

Correspondence: Dr. T. Sawada, Department of Oral Pathology, Tokyo Dental College, Masago 1-2-2, Mihama-ku, Chiba-city, Chiba 261, Japan. basement membrane. As of yet, however, only a limited number of reports $(1,8,12)$ have demonstrated laminin and type-IV collagen in the tooth germ at the ultrastructural level. The purpose of the present study is to perform immuno-electron microscopic investigations to localize laminin and type-IV collagen in basement membranes obtained from different regions of the continuously forming incisors of young rats.

\section{MATERIALS AND METHODS}

Young male Wistar rats, weighing about $100 \mathrm{~g}$ 
each, were anesthetized with ketamine hydrochloride $(10 \mathrm{mg} / \mathrm{kg}$, i.p. $)$ and fixed by perfusion with a $0.05 \%$ saponin-supplemented mixture of periodate lysine and a $4 \%$ paraformaldehyde solution (11) for $20 \mathrm{~min}$ at $4^{\circ} \mathrm{C}$. After perfusion, the upper incisors were dissected out and placed in the same fixative for $6 \mathrm{hr}$ at $4^{\circ} \mathrm{C}$. They were then demineralized in $10 \%$ EDTA for 3 weeks at $4^{\circ} \mathrm{C}$. After demineralization, the specimens were rinsed in increasing concentrations of sucrose in $0.01 \mathrm{M}$ phosphate-buffered saline (PBS), embedded in Tissue Tek OCT Compound (Miles Laboratories, Inc., Napervill, Illinois), and prepared into longitudinal frozen sections of $6 \mu \mathrm{m}$ thick prepared in a cryostat. A schematic diagram (19) of a young rat upper incisor is shown in Fig. 1.

The frozen sections were immersed in either 5\% normal goat serum or $5 \%$ normal rabbit serum diluted in PBS for a few min to block nonspecific reactions of the antibodies that were to be used for immuno-staining. The sections were then incubated with primary antibodies (rabbit anti-laminin antisera, BRL, Life Technol., Bethesda, diluted $1: 200$ in PBS; goat antitype-IV-collagen antibody, Southern Biotechnology Associates, Inc., Alabama, diluted $1: 200$ in PBS) for $16 \mathrm{hr}$ at $4^{\circ} \mathrm{C}$ in a moist chamber. The purity, specificity and characteristic interspecies cross-reactivities of the antibodies used in the present study have been described previously $(13,14,21)$. Sections used as controls were incubated in the same manner with normal rabbit serum, normal goat serum, or PBS only.
After being rinsed in PBS, they were incubated with secondary antibodies (horseradish peroxidase-labeled goat anti-rabbit IgG; horseradish peroxidase-labeled rabbit anti-goat IgG) for $16 \mathrm{hr}$ at $4^{\circ} \mathrm{C}$. After incubation, the sections were fixed with $0.5 \%$ glutaraldehyde in $0.01 \mathrm{M}$ PBS for $5 \mathrm{~min}$, rinsed in PBS, preincubated in $0.02 \% 3,3^{\prime}$-diaminobenzidine solution for $30 \mathrm{~min}$, and then incubated in a diaminobenzidine solution containing $1.6 \mathrm{mM}$ hydrogen peroxide (5) for $5 \mathrm{~min}$ at room temperature. The sections were postfixed with $2 \%$ osmium tetroxide in a $0.1 \mathrm{M}$ sodium-phosphate buffer for $1 \mathrm{hr}$, embedded in epoxy resin (Quetol 812, Nisshin EM, Tokyo). The ultrathin sections were cut, and observed in an electron microscope (H-600, Hitachi, Tokyo) at $75 \mathrm{kV}$ without staining.

\section{RESULTS}

In the growing end region of the rat-incisor enamel organ (Fig. 1), immunoreaction products for laminin and type-IV collagen were observed in the basement membrane and the fibrillar layer attached to it (Figs. 2a, b). This layer demarcated the inner enamel epithelium from the dental papilla. Coated pits appearing in the plasma membranes of the distal ends of inner enamel epithelium cells contained reaction products (Figs. 2a, b). Occasionally reaction products for laminin and type-IV collagen were found in the rough endoplasmic reticulum cisternae of inner enamel epithelium cells (Fig. 2c). In dental papillae,

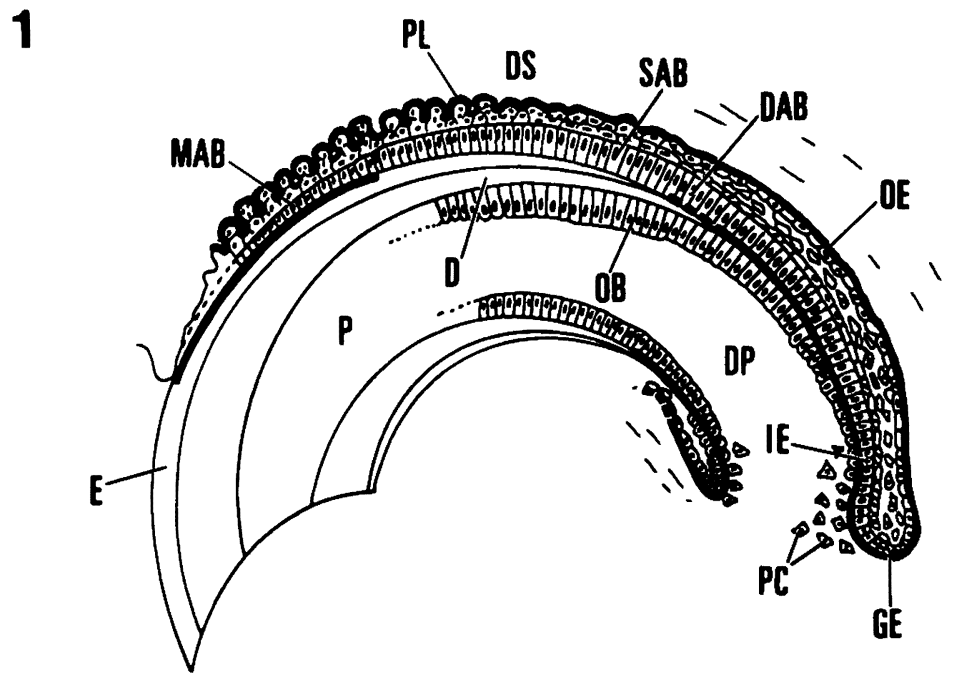

Fig. 1. Longitudinal section of an upper incisor from a young rat. Bold lines indicate distribution of the basement membrane at different stages of odontogenesis. D: dentin, DAB: differentiating ameloblasts, DP: dental papilla, DS: dental sac, E: enamel, GE: growing end, IE: inner enamel epithelium, MAB: maturing ameloblasts, OB: odontoblasts, OE: outer enamel epithelium, P: dental pulp, PC: dental papilla cells, PL: papillary layer, SAB: secretory ameloblasts. 


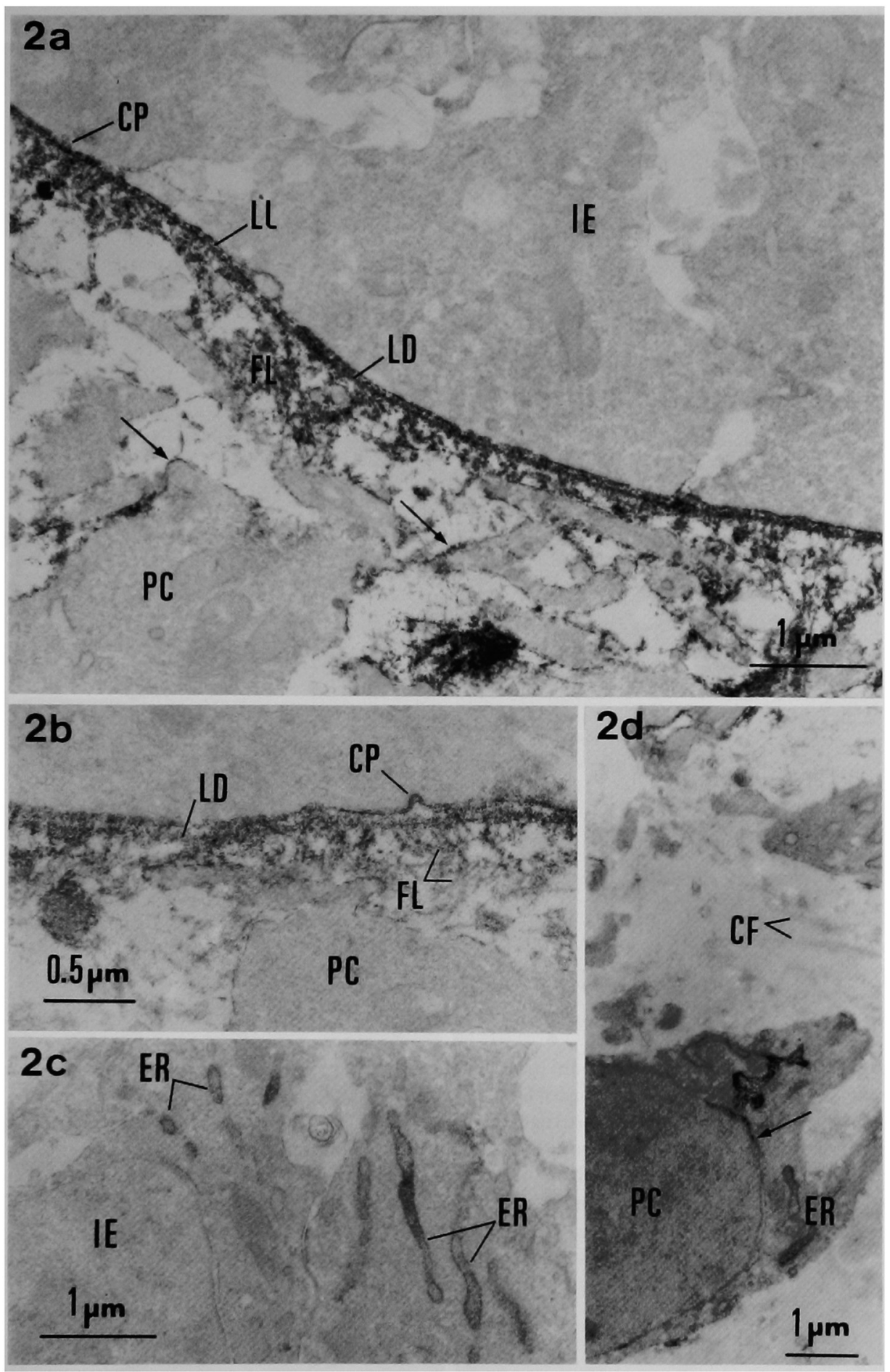

Fig. 2a. Localization of laminin. Immunoreaction products are seen in the lamina lucida (LL), lamina densa (LD), and fibrillar layer (FL). Reaction products (arrows) are also located on the external surface of the dental papilla cells (PC). CP: coated pit, IE: inner enamel epithelium cell.

FIG. 2b. Localization of type-IV collagen. The fibrillar layer (FL) reacts positively for type-IV collagen. LD: lamina densa, CP: coated pit, PC: dental papilla cell.

Fig. 2c. Localization of type-IV collagen. Reaction products occur in the rough endoplasmic reticulum cisternae (ER) of the inner enamel epithelium cell (IE).

Fic. 2d. Localization of laminin. Reaction products are seen in the cisternae of rough endoplasmic reticulum (ER) and perinuclear spaces (arrow) of the dental papilla cell (PC). CF: collagen fibrils. 


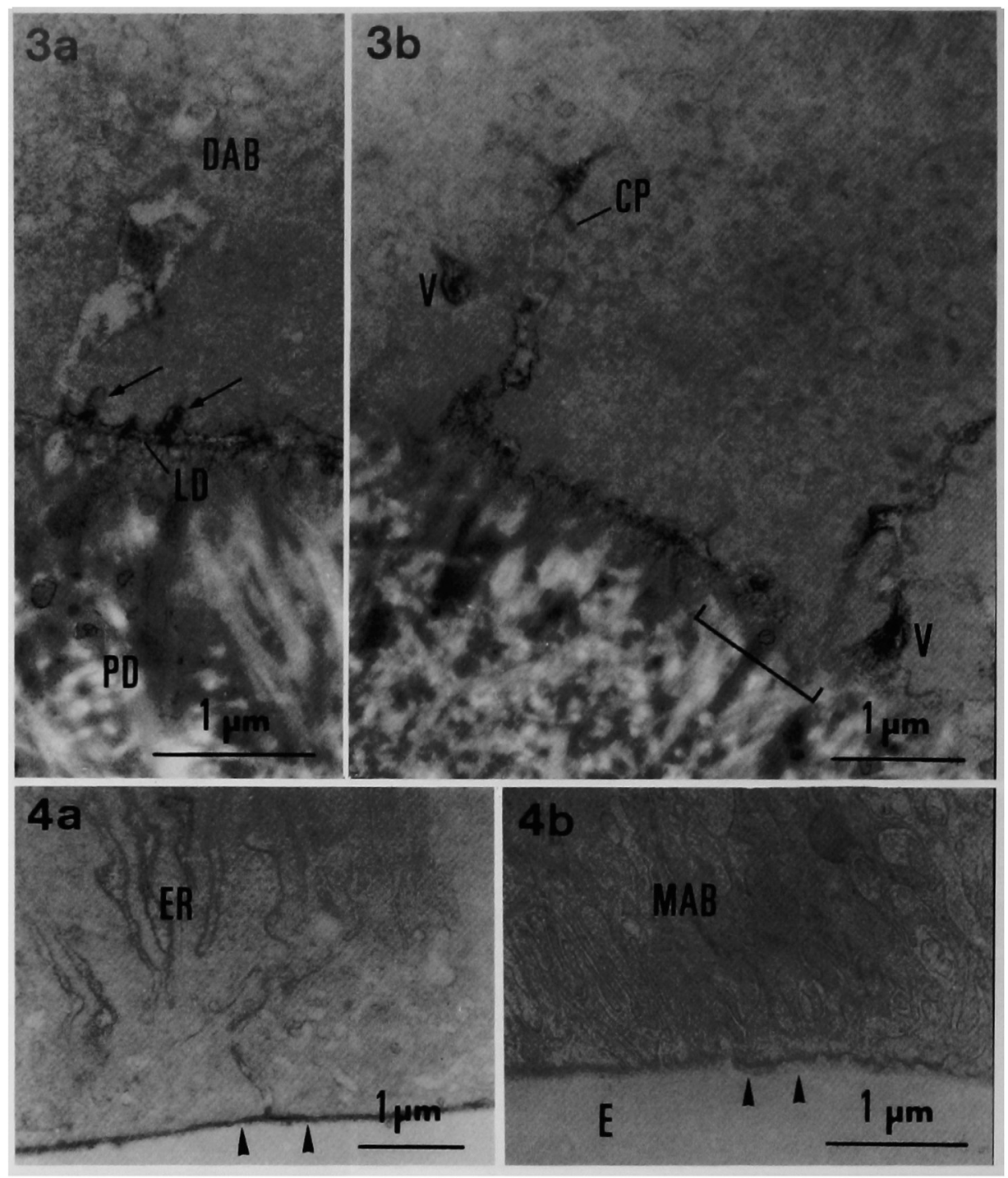

FIG. 3a. Localization of laminin. Irregularly undulating distal plasma membranes form minute invaginations containing reaction products (arrows). DAB: differentiating ameloblast, LD: lamina densa, PD: predentin.

FIG. 3b. Localization of type-IV collagen. Deep, irregular invaginations (V) and coated pits (CP) contain positive reaction products. The basement membrane demonstrates weak and discontinuous immunoreactivity ( $\sqcup)$.

FIG. 4a. Localization of laminin. On the enamel surface, a structure resembling the basement membrane shows reaction products (arrowheads). Reaction products may be observed in the cisternae of the rough endoplasmic reticulum (ER) of the maturing ameloblast.

Fig. 4b. Localization of type-IV collagen. No immunoreaction products are detectable in the basement membrane-like structure (arrowheads). MAB: maturing ameloblasts. E: enamel space.

Fig. 5a. Localization of laminin. Continuous immunoreactions are seen in the basement membrane (BM) of the outer enamel epithelium (OE). Note reaction products within the cisternae of the rough endoplasmic reticulum (ER) and in the perinuclear spaces (arrows) of the cells. EC: endothelial cell.

Frg. 5b. Localization of type-IV collagen. Reaction products are frequently seen in the rough endoplasmic reticulum (ER) of the outer enamel epithelial cells (OE). BM: basement membrane, DS: dental sac.

Fig. 5c. Localization of laminin. The outer enamel epithelial cells (OE) located above the secretory ameloblasts exhibit weak reaction in the rough endoplasmic reticulum (ER). 


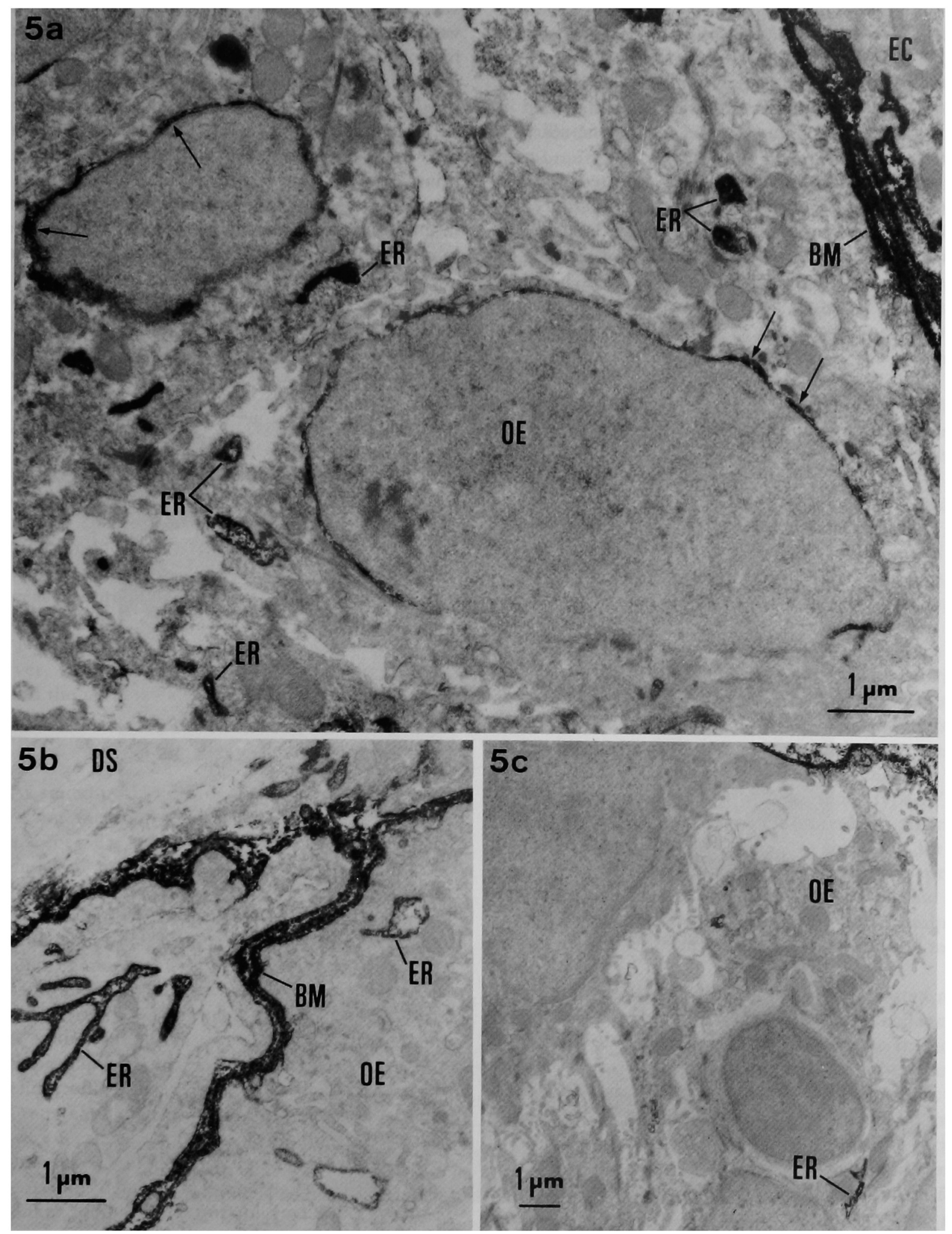


the external surfaces of differentiating dental papilla cells were outlined by reaction products (Figs. 2a, b). Reaction products were occasionally observed in cisternae of rough endoplasmic reticulum and in perinuclear spaces in dental papilla cells (Fig. 2d). The collagen fibrils that were randomly distributed in the dental papillae demonstrated no specific reaction products (Fig. 2d).

In differentiating ameloblasts located on the predentin (Fig. 1), plasma membrane undulation (Fig. $3 a)$ in the distal ends resulted in deep, irregular invaginations (Fig. $3 \mathrm{~b}$ ). Various numbers of coated pits were observed in the plasma membrane in these invaginations. Reaction products occurred in both invaginations and coated pits (Figs. $3 a, b$ ). Immunoreactivity in the basement membranes of differentiating ameloblasts was consistently lower than that of the inner enamel epithelium. Reactions gradually became indistinct (Fig. 3a) and discontinuous (Fig. $3 \mathrm{~b}$ ) and disappeared completely when sporadic mineralization occurred in the predentin.

In maturing ameloblasts (Fig. 1), structures resembling the basement membrane, composed of a lamina lucida and a lamina densa, and lacking a

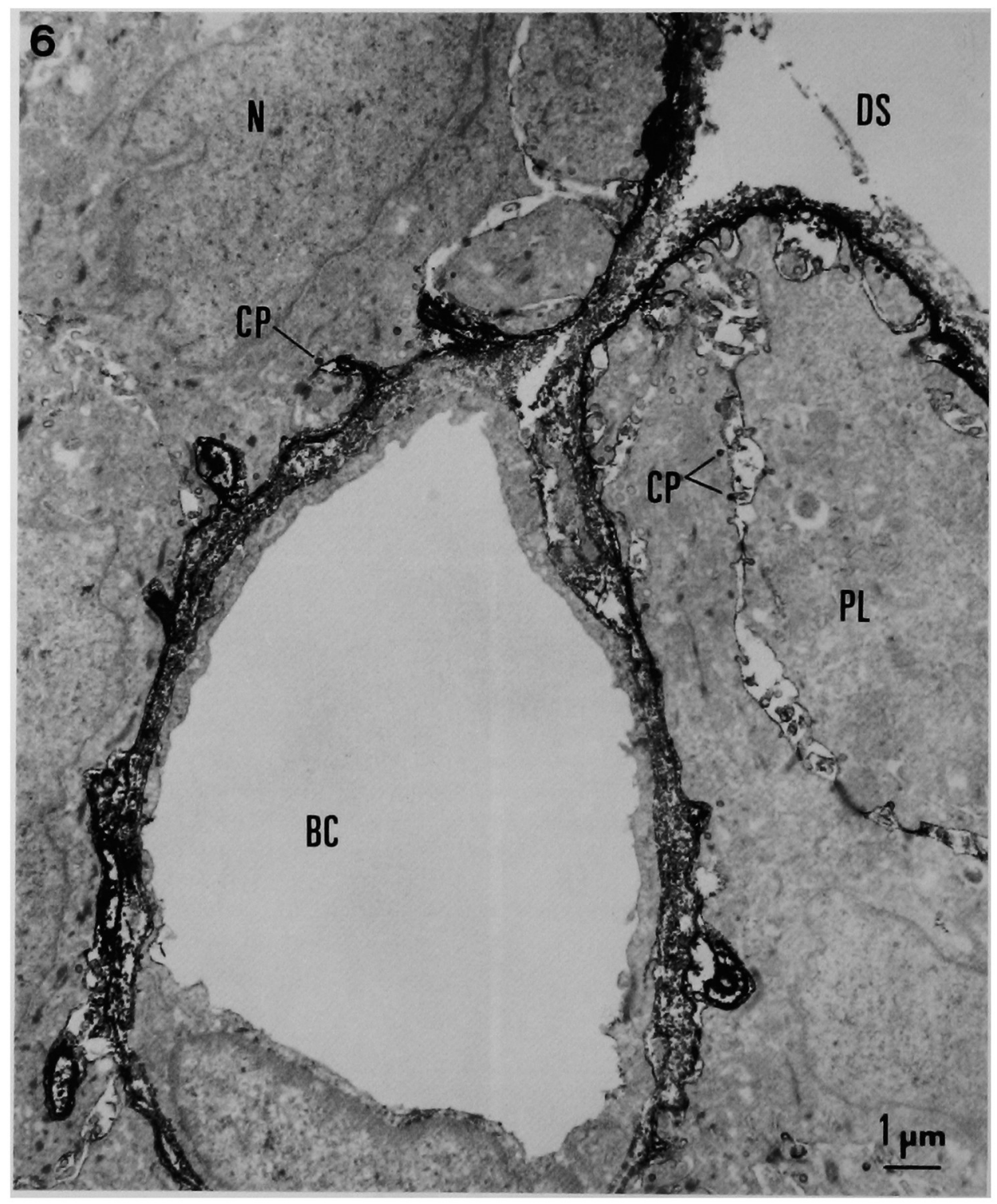

FIG. 6. Localization of laminin. Intense reaction is seen in the basement membranes of both the papillary layer (PL) and the blood capillary (BC). Coated pits (CP) also react positively. DS: dental sac, N: nucleus of a papillary layer cell. 
fibrillar layer occurred between the distal ends of cells and the surface of the enamel. Reaction products for laminin antisera were found in this basement membrane-like structure (Fig. 4a), which, however, lacked reaction products for type-IV collagen (Fig. 4b). Reaction products for laminin were occasionally seen in cisternae in the rough endoplasmic reticulum of maturing ameloblasts (Fig. 4a).

Immunoreaction for laminin and type-IV collagen was observed in the basement membrane demarcating the outer enamel epithelium (Figs. 1, 5a, b) and the papillary layer (Figs. 1, 6) from the dental sac. Reaction was also observed in the basement membrane of blood capillaries that had penetrated into the papillary layer (Fig. 6). Reaction products were frequently observed in the perinuclear spaces and rough endoplasmic reticulum cisternae of the outer enamel epithelial cells and in the endothelial cells of blood capillaries in the dental sac (Figs. 5a, b). Furthermore, toward the incisal end from the stage of matrix formation, a decreasing reaction gradient was noted in the rough endoplasmic reticulum cisternae and the perinuclear spaces (compare Fig. 5a with Fig. 5c). Reaction products were observed in the plasma membranes of papillary layer cells (Fig. 6). In the coated pits, reaction products frequently occurred in distal or lateral plasma membranes of papillary layer cells (Fig. 6). No definite reaction products were observed, however, in the rough endoplasmic reticulum cisternae and the perinuclear spaces of these cells (Fig. 6).

\section{DISCUSSION}

As stated in the introduction, neither the precise nature nor the origin of the dental basement membrane, especially the fibrils attached to it, has been elucidated in previous works $(2,3,15,16,22,24$, $25)$. The present study clearly detected reaction products to both laminin and type-IV collagen, two major components of the basement membrane $(6,7,10)$, in the inner enamel epithelium basement membrane as well as in the fibrillar layer attached to it. The occasional occurrence of immunoreaction products in the rough endoplasmic reticulum (secretory and membrane protein generating sites) of inner enamel epithelium cells indicates production of the basement membrane components. In addition, reaction products were localized on the outer cell membranes and in the rough endoplasmic reticulum cisternae of differentiating dental papilla cells. Our recent work on monkey tooth germs (unpublished data) produced similar results, which suggest that inner enamel epithelial cells and dental papilla cells are involved simultaneously in the formation of the fibrillar layer. This idea may be supported by recent immunofluorescence studies indicating that interaction of epithelial and mesenchymal cells is essential to basement membrane formation $(23,27)$.

Previous studies of both human (28) and monkey (18) tooth germs have indicated that the basement membrane and the fibrillar layer attached to it are engulfed and degraded by differentiating ameloblasts. The results of the present study confirm this view by providing additional evidence of the presence of laminin and type-IV collagen in the coated pits and deep invaginations that, together, form an engulfing system on the surfaces of differentiating ameloblasts. Reconfirming the idea just mentioned, in recent simultaneous immuno- and enzymecytochemical work, we have shown that acid phosphatase positive granules, present in inner enamel epithelial cells, increase in number and sometimes appear in proximity to deep invaginations in differentiating ameloblasts (20).

This study did not elucidate the precise nature of the basement membrane-like structure which appeared between the maturing ameloblasts and the enamel surface (Fig. 4a). Reaction products for laminin antisera were localized in this structure and in rough endoplasmic reticulum cisternae of the ameloblasts. The reason for the absence of type-IVcollagen staining in this structure remains unclear, although it may be related to a complete hindrance of the antigenic exposure of type-IV collagen by other molecules (blocking) or perhaps to a lack of the antigen (17). It could be another possibility that the immunogenicity required for the detection of type-IV collagen in the basement membrane-like structure decreases easily during tissue preparation: for instance, during the EDTA demineralization procedure. On the basis of our results, it can be said that the structure resembling the basement membrane differs in nature from other dental basement membranes.

The present study frequently demonstrated reaction products to both laminin and type-IV collagen in the rough endoplasmic reticulum cisternae including the perinuclear spaces of outer enamel epithelial cells. It may be assumed, therefore, that both laminin and type-IV collagen are actively synthesized by outer enamel epithelial cells in the basal area of the incisor. This phenomenon may account for the production, in large quantities, of the components required to form the papillary layer basement membrane and may result in an enlargement of surface areas coming into contact with the dental sac and its capillaries. The 
lack of definite reaction products in these organelles of the papillary layer cells may be explained either by extremely slow turnover rate and synthesis of these two substances, as has been suggested by others (12), or by the cessation of their production. The precise stage at which cells cease such production, however, remains unsolved.

Taken together, these results indicate that dental basement membranes are dynamically metabolized during tooth formation. Although its exact function is unknown, the basement membrane in the rat incisor may be involved directly or indirectly in the control of tooth formation.

\section{ACKNOWLEDGEMENTS}

We wish to thank Mr. H. Suzuki for photographic assistance and to Mr. R. L. Gage for his help in correcting English. This work was supported in part by a Grant-in-Aid for scientific research from the Ministry of Education, Science, and Culture of Japan.

\section{REFERENCES}

1. Andujar, M. B., Magloire, H., Hartmann, D. J., Ville, G. and Grimaud, J. A.: Early mouse molar root development: Cellular changes and distribution of fibronectin, laminin and type-IV collagen. Differentiation 30; 111-122, 1985.

2. Casasco, A.: Ultrastructural study of the rat enamel organ and adjoining extracellular matrix during early tooth development. Bull. Group. int. Rech. sc. Stomat. et Odont. 30; 101-112, 1987.

3. Egawa, I.: Electron microscopy of human enamel organ. Shikwa Gakuho 70; 803-836, 1970.

4. Frank, R. M. and Nalbandian, J.: Development of dentine and pulp. In "Handbook of Microscopic Anatomy", Vol. V/6 Teeth, ed. by A. Oksche and L. Vollrath, Springer-Verlag, Berlin, 1989, pp. 73-171.

5. Graham, Jr., R. C. and Karnovsky, M. J.: The early stages of absorption of injected horseradish peroxidase in the proximal tubules of monkey kidney: Ultrastructural cytochemistry by a new technique. J. Histochem. Cytochem. 14; 291-302, 1966.

6. Kefalides, N. A.: Structure and biosynthesis of basement membranes. Int. Rev. Connect. Tissue Res. 6; 63-104, 1973.

7. Kleinman, H. K., Cannon, F. B., Laurie, G. W., Hassell, J. R., Aumailley, M., Terranova, V. P., Martin, G. R. and DuBois-Dalcq, M.: Biological activities of laminin. J. Cell Biochem. 27; 317-325, 1985.

8. Laurie, G. W., Leblond, C. P. and Martin, G. R.: Localization of type-IV collagen, laminin, heparan sulfate proteoglycan, and fibronectin to the basal lamina of basement membranes. J. Cell Biol. 95; 340-344, 1982.

9. Lesot, H., Osman, M. and Ruch, J. V.: Immunofluorescent localization of collagens, fibronectin, and laminin during terminal differentiation of odontoblasts. Develop. Biol. 82; 371-381, 1981.

10. Martin, G. R. and Timpl, R.: Laminin and other basement membrane components. Annu. Rev. Cell Biol. 3; 5785, 1987.

11. McLean, I. W. and Nakane, P. K.: Periodate-lysineparaformaldehyde fixative. A new fixative for immunoelectron microscopy. J. Histochem. Cytochem. 22; 1077-1083, 1974.

12. Murray, I. C. and Leblond, C. P.: Immunoelectron microscopy of endothelial cells in rat incisor suggests that most basement membrane components are produced by young cells, whereas heparan sulfate proteoglycan is produced by both young and old cells. $J$. Histochem. Cytochem. 36; 763-773, 1988.

13. Nakamura, S. and Fujimori, O.: Immunohistochemical studies on the connective tissues of the salivary grands of the rat in postnatal development. J. Nagoya City University Medical Association 42; 517-540, 1991.

14. Nakanishi, Y., Nogawa, H., Hashimoto, Y., Kishi, J. and Hayakawa, T.: Accumulation of collagen III at the cleft points of developing mouse submandibular epithelium. Development 104; 51-59, 1988.

15. Orams, H.J.: The ultrastructure of tissues at the epithelial-mesenchymal interface in developing rat incisors. Arch. Oral Biol. 23; 39-44, 1978.

16. Reith, E. J.: The early stage of amelogenesis as observed in molar teeth of young rats. J. Ultrastruct. Res. 17; 503526, 1967.

17. Salonen, J. and Santii, R.: Ultrastructural and immunohistochemical similarities in the attachment of human oral epithelium to the tooth in vivo and to an inert substrate in an explant culture. J. Periodont. Res. 20; 176-184, 1985.

18. Sawada, T., Yanagisawa, T. and Takuma, S.: Epithelial-mesenchymal junctional area in an early stage of odontogenesis in Macaca fuscata. Adv. Dent. Res. 1; 141$147,1987$.

19. Sawada, T., Yamamoto, T., Yanagisawa, T., Takuma, S., Hasegawa, $H$. and Watanabe, $K$.: Immunohistochemistry of the distribution of $\alpha$-tubulin in rat-incisor odontoblasts and ameloblasts. Acta Histochem. Cytochem. 22; 617-624, 1989.

20. Sawada, T., Yamamoto, T., Yanagisawa, T., Takuma, S., Hasegawa, H. and Watanabe, K.: Evidence for uptake of basement membrane by differentiating ameloblasts in the rat incisor enamel organ. J. Dent. Res. 69; 1508-1511, 1990.

21. Schiff, R. and Rosenbluth, J.: Ultrastructural localization of laminin in rat sensory ganglia. $J$. Histochem. Cytochem. 34; 1691-1699.

22. Silva, D. G. and Kailis, D. G.: Ultrastructural studies on the cervical loop and the development of the amelo-dentinal junction in the cat. Arch. Oral Biol. 17; 279-289, 1972.

23. Simon-Assmann, P., Bouziges, F., Arnold, C., Haffen, 
K. and Kedinger, M.: Epithelial-mesenchymal interactions in the production of basement-membrane components in the gut. Development 102; 339-347, 1988.

24. Skobe, Z., Stern, D. and Prostak, K.: Ultrastructure of differentiating preameloblasts from tooth germs of the permanent dentition of Macaca mulatta and Macaca arctoides. Calcif. Tissue Int. 33; 603-618, 1981.

25. Takuma, S.: Ultrastructure of dentinogenesis. In "Structural and Chemical Organization of Teeth", Vol. 1, ed. by A. E. W. Miles, Academic Press, New York, 1967, pp. 325-370.
26. Thesleff, I., Stenman, S., Vaheri, A. and Timpl, R.: Changes in the matrix proteins, fibronectin and collagen, during differentiation of mouse tooth germ. Develop. Biol. 70; 116-126, 1979.

27. Xu, Z., Parker, S. B. and Minkoff, R.: Influence of epithelial-mesenchymal interaction on the viability of facial mesenchyme. II: Synthesis of basement-membrane components during tissue recombination. Anat. Rec. 228; 58-68, 1990.

28. Yama, K.: Ultrastructure of human ameloblasts. Shikwa Gakuho 71; 1531-1574, 1971. 